\title{
On the Largest Minorants Associated with a Matrix-Valued Caratheodory Function and Spectral Factorization
}

\author{
B. FRITZSCHE and B. KIRSTEIN
}

\begin{abstract}
We will study various aspects of largest minorants associated with matrix-valued Carathéodory functions in the context of spectral factorization. The first main aim is to derive explicit interrelations between the largest minorants associated with a Cayley-linked pair consisting of a matrix-valued Caratheodory function and a matrix-valued Schur function. The second goal is to derive corresponding factorizations for the normalized limit semi-radius functions associated with a matrix-valued Carathéodory function having finite entropy.
\end{abstract}

Key words: Carathéodory functions, largest minorants, Weyl matrix balls

AMS subject classification: $30 \mathrm{E} 05$

\section{Introduction}

The classes of left and right maximal matrix-valued functions originate in prediction theory of multivariate stationary sequences. WIENER and MASANI [26] created the notion of the generating function of a multivariate stationary sequence. This matrixvalued function turned out to be an essential object in prediction'theory. MASANI [19], [20] studied the analytic properties of these generating functions. In particular, he recognized that such functions are so-called left maximal functions belonging to the matricial Hardy class, which can be conceived as an immediate matricial generalization of scalar outer functions belonging to the ordinary Hardy class. The importance of maximal functions is essentially caused by their role as largest minorants of a Lebesgue-integrable nonnegative Hermitian matrix-valued function on the unit circle (see MASANI [19], [20], SZ.-NAGY and FOIAS [25], SUCIU and VALUSESCU [24]; CONSTANTINESCU [5] and BAKONYI [3]).

In view of famous theorems due to F.Riesz-Herglotz and Kolmogorov, there is an essentially unique correspondence between multivariate stationary sequences and matrixvalued Carathéodory functions. In this paper, we will study certain properties of largest minorants associated with matrix-valued Carathéodory functions. Parts of this paper con-

B. Fritzsche: Universität Leipzig, Institut für Mathomatik, Augustuspl. 10, D - 04109 Leipzig B. Kirstein: Universität Leipzig. Institut für Mathematik. Augustuspl. 10, D - 04109 Leipzig 
tain a continuation of the authors' former studies on the Weyl matrix balls associated with nondegenerate matrix-valued Caratheodory functions (see [11, Parts IV and V], [13]). For prediction theoretical interpretations of these Weyl matrix balls, we refer the reader to the papers [11].

Factorizations of the normalized limit semi-radius functions generated by a sequence of nested Weyl matrix balls were first studied by DUBOVOJ [7] in connection with nondegenerate matrix-valued Schur functions. Using a theorem due to BRODSKII [4] he represented such a nondegenerate Schur function $f$ as the characteristic operator function of an appropriately chosen unitary colligation $\Delta$. With the aid of this $\Delta$ Dubovoj defined certain matrix-valued functions $\varphi$ and $\psi$ and formulated the assertion that $\varphi \varphi^{*}$ and $\psi^{*} \psi$ are exactly the normalized left and right semi-radius functions associated with the given $f$. However, his proof given in [7] was not correct. Influenced by DUBOVOJ's paper [7], KATSNELSON [16] studied the normalized limit semi-radius functions occurring in the matricial version of KREIN's [18] continuation problem for positive definite functions given on a finite interval of the real axis. Katsnelson's approach is based on two essential components, namely on the classical work of HELSON and LOWDENSLAGER [15] on prediction theory of stationary sequences and on fundamental results in weighted approximation (see ACHIESER [1], MERGELJAN [21]). In particular, Katsnelson recognized that the normalized limit semi-radius functions generated by the sequence of (nested) Weyl matrix balls occurring in the matrix version of Krein's problem can be written as $\Phi \Phi^{*}$ and $\Psi^{*} \Psi$ where $\Phi$ and $\Psi$ are outer matrix-valued functions in the upper half plane.

Inspired by DUBOVOJ's [7] and KATSNELSON's [16] investigations the authors [11, Part IV] turned their attention to the normalized left and right limit semi-radius functions associated with a matrix-valued Schur function having finite entropy. Using results due to DELSARTE, GENIN and KAMP [6] on the asymptotic behaviour of matrix polynomials orthogonal with respect to a given nonnegative Hermitian-valued Borel measure on the unit circle, we recovered Dubovoj's factorization theorem. Moreover, we recognized that these factorizations are realized by outer matrix-valued functions in the unit disc. It should be mentioned that, during his stay in Leipzig in 1990, Dubovoj informed the authors that he found a correct proof of his original result. His proof is based on his joint paper [9] with RAMADAN K. MOHAMMED, where BRODSKII's [4] generalization of the known SZ.-NAGY-FOIAS model for contractions [25] is extensively used.

One of the main aims of this paper is to derive corresponding factorizations for the normalized limit semi-radius functions associated with a matrix-valued Carathéodory function having finite entropy. Our approach relies heavily on the asymptotic behaviour of matrix polynomials orthogonal with respect to the F.-Riesz-Herglotz measure associated with the considered matrix-valued Caratheodory function.

- Another main goal of this paper is the study of further aspects of spectral factorization. More precisely, we will derive explicit interrelations between the largest minorants associated with a Cayley-linked pair consisting of a matrix-valued Carathéodory function and a matrix-valued Schur function. Moreover, we will discuss connections between the largest minorants associated with a nonsingular matrix-valued Carathéodory function $\Omega$ and its inverse $\Omega^{-1}$. Hereby, we will essentially use the maximum modulus principle for the Smirnov class. 


\section{Preliminaries}

Let us begin with some notations and preliminaries. Throughout this paper, let $m$, $p$ and $q$ be positive integers. We will use $\mathbb{N}_{0}$ and $\mathbb{C}$ to denote the set of all nonnegative integers and the set of all complex numbers, whereas $\mathbb{D}, \mathbb{T}$ and $\mathbb{C}_{0}$ stand for the open unit disc, the unit circle and the extended complex plane, respectively:

$$
\mathbb{D}:=\{z \in \mathbb{C}:|z|<1\}, \mathbb{T}:=\{z \in \mathbb{C}:|z|=1\}, \mathbb{C}_{0}:=\mathbb{C} \cup\{\infty\}
$$

The linear Lebesgue-Borel measure on $\mathbb{T}$ will be designated by $\tilde{\lambda}$.

If $\chi$ is a nonempty set, then $\chi^{p \times q}$ denotes the set of all $p \times q$ matrices each entry of which belongs to $\chi$. The symbol $0_{p \times q}$ stands for the null matrix that belongs to $\mathbb{C}^{p \times q}$, whereas $I_{p}$ designates the identity matrix which belongs to $\mathbb{C}^{p \times p}$. In cases where the size of the null matrix (respectively, the identity matrix) is clear, we will omit the indexes. If $A$ and $B$ are $p \times p$ Hermitian matrices, the Löwner semi-ordering $A \geqq B$ means that $A-B$ is nonnegative Hermitian. If $A-B$ is positive Hermitian, then we will also write $A>B$ to indicate this fact. If $A \in \mathbb{C}^{p \times q}$, then the symbol $\|A\|$ designates its norm as an operator from $\mathbb{C}^{q}$ into $\mathbb{C}^{p}$ when both of these spaces are equipped with the standard inner product. If $A$ belongs to $\mathbb{C}^{p \times p}$, then the Hermitian matrices

$$
\operatorname{Re} A:=\frac{1}{2}\left(A+A^{*}\right) \text { and } \operatorname{Im} A:=\frac{1}{2 i}\left(A-A^{*}\right)
$$

are called the real part of $A$ and the imaginary part of $A$, respectively. If $A \in \mathbb{C}^{p \times p}$ satisfies $\operatorname{Re} A \geqq 0$, then it is readily checked that $\operatorname{det}(I+A) \neq 0$. then

If $\mathfrak{B}$ stands for the complex linear space of all Borel measurable functions $f: \mathbf{T} \rightarrow \mathbb{C}^{p \times q}$,

$$
\mathcal{3}:=\left\{f \in \mathfrak{B}: \tilde{\lambda}\left(\left\{\zeta \in \mathbf{T}: f(\zeta) \neq 0_{p \times q}\right\}\right)=0\right\}
$$

is a linear subspace of $\mathfrak{B}$. If $f \in \mathfrak{B}$, then we will use $\langle f\rangle$ to indicate the element of the quotient space $\mathfrak{B} / \mathfrak{Z}$ which is generated by $f$. Obviously, $\langle f\rangle=\langle g\rangle$ if and only if $f(\zeta)=g(\zeta)$ for $\tilde{\lambda}$-almost all $\zeta \in \mathbb{T}$.

Let $\mathfrak{B}_{p \times q}$ be the $\sigma$-algebra of Borelian subsets of $\mathbb{C}^{p \times q}$. If $(\Lambda, \mathfrak{A}, \mu)$ is a measure space and if $t \in(0, \infty)$, then let $\mathfrak{L}^{t}(\Lambda, \mathfrak{A}, \mu ; \mathbb{C})$ be the set of all $\mathfrak{A}-\mathfrak{B}_{1 \times 1}$-measurable functions $f: \Lambda \rightarrow \mathbb{C}$ such that $|f|^{t}$ is integrable with respect to the measure $\mu$, whereas $\mathfrak{L}^{\infty}(\Lambda, \mathfrak{A}, \mu ; \mathbb{C})$ stands for the set of all essentially bounded $\mathfrak{A}-\mathfrak{B}_{1 \times 1}$ measurable functions $f: \Lambda \rightarrow \mathbb{C}$. It is well-known that $\left[\mathfrak{L}^{t}(\Lambda, \mathfrak{A}, \mu ; \mathbb{C})\right]^{p \times q}$ coincides with the set of all $\mathfrak{A}-\mathfrak{B}_{p \times q}$-measurable functions $f: \Lambda \rightarrow \mathbb{C}^{p \times q}$ for which $\left(\operatorname{tr} f^{*} f\right)^{t / 2}$ is integrable with respect to $\mu$.

Lemma 1: Let $(\Lambda, \mathfrak{A}, \mu)$ a measure space, and let $W \in\left[\mathcal{L}^{1}(\Lambda, \mathfrak{L}, \mu ; \mathbb{C})\right]^{p \times p}$.

(a) If $g: \Lambda \rightarrow \mathbb{C}^{p \times q}$ is an $\mathfrak{A}-\mathfrak{B}_{p \times q}$-measurable function which satisfies $g g^{*} \leq W \mu-$ a.e. on $\Lambda$, then $g$ belongs to $\left[\mathfrak{L}^{2}(\Lambda, \mathfrak{A}, \mu ; \mathbb{C})\right]^{p \times q}$.

(b) If $h: \Lambda \rightarrow \mathbb{C}^{q \times p}$ is an $\mathfrak{A}-\mathfrak{B}_{p \times q}$-measurable function which satisfies $h^{*} h \leqq W \mu$. a.e. on $\Lambda$, then $h$ belongs to $\left[\mathfrak{L}^{2}(\Lambda, \mathfrak{A}, \mu ; \mathbb{C})\right]^{p \times q}$.

The proof of Lemma 1 is straightforward. We omit the details. 


\section{Some Facts on Various Classes of Meromorphic Matrix-valued Functions}

First we will summarize some basic facts on particular classes of meromorphic functions. A detailed treatment of this subject can be found, e.g., in NEVANLINNA [22] and DUREN [10].

Suppose that $G$ is a simply connected domain of $\mathbb{C}_{0}$. Then let $\mathcal{N} \mathcal{M}(G)$ be the Nevanlinna class of all functions which are meromorphic in $G$ and which can be represented as quotient of two bounded holomorphic functions in $G$. Observe that $\mathcal{N} \mathcal{M}(G)$ turns out to be a division algebra over $\mathbb{C}$. If $g \in \mathcal{N} \mathcal{M}(\mathbb{D})$, then a well-known theorem due to Fatou implies that there exist a Borelian subset $\mathfrak{B}_{0}$ of the unit circle $\mathbf{T}$ with $\tilde{\lambda}\left(\mathfrak{B}_{0}\right)=0$ and a Borel measurable function $\underline{g}: \mathbf{T} \rightarrow \mathbb{C}$ such that

$$
\lim _{r \rightarrow 1-0} g(r z)=\underline{g}(z)
$$

for all $z \in \mathbf{T} \backslash \mathfrak{B}_{0}$. In the following, we will continue to use the symbol $g$ to denote the boundary function of a function $g$ which belongs to $\mathcal{N} \mathcal{M}(\mathbb{D})$. The subalgebra of all $g \in \mathcal{N} \mathcal{M}(G)$ which are holomorphic in $G$ will be designated by $\mathcal{N}(G)$. The class $\mathcal{N}(\mathbb{D})$ can be characterized as the set of all functions $g$. which are holomorphic in $\mathbb{D}$ and which satisfy

$$
\sup _{r \in[0,1)} \frac{1}{2 \pi} \int_{\mathbf{T}} \log ^{+}|g(r z)| \tilde{\lambda}(d z)<+\infty
$$

where $\log ^{+} x:=\max (\log x, 0)$ for each $x \in[0, \infty)$.

If a function $g: \mathbb{D} \rightarrow \mathbb{C}$ admits a representation

$$
g(w)=\alpha \cdot \exp \left[\frac{1}{2 \pi} \int_{\mathbf{T}} \frac{z+w}{z-w} \log k(z) \tilde{\lambda}(d z)\right], \quad w \in \mathbb{D},
$$

with some $\alpha \in \mathbb{T}$ and some Borel measurable function $k: \mathbb{T} \rightarrow[0, \infty)$ which satisfies

$$
\frac{1}{2 \pi} \int_{\mathbf{T}}|\log k| \dot{d} \tilde{\lambda}<+\infty
$$

then $g$ belongs to $\mathcal{N}(\mathbb{D})$. Such functions are called outer functions in $\mathcal{N}(\mathbb{D})$. If $g \in \mathcal{N}(\mathbb{D})$ is outer, then $|\underline{g}|=k$ a.e. on $\mathbf{T}$.

For each $g \bar{\in} \mathcal{N}(\mathrm{D})$, the inequality

$$
\frac{1}{2 \pi} \int_{\mathbf{T}} \log ^{+}|g(z)| \tilde{\lambda}(d z) \leqq \lim _{r \rightarrow 1-0} \frac{1}{2 \pi} \int_{\mathbf{T}} \log ^{+}|g(r z)| \tilde{\lambda}(d z)
$$

is satisfied. By the Smirnov class $\mathcal{N}_{+}(\mathrm{ID})$ we will mean the set of all $g \in \mathcal{N}(\mathbb{D D})$ for which equality holds true in (1). The class $\mathcal{N}_{+}(\mathrm{DD})$ proves to be a subalgebra of $\mathcal{N}(\mathrm{ID})$. If $g$ is outer in $\mathcal{N}(\mathbb{D})$, then $g$ necessarily belongs to $\mathcal{N}_{+}$(ID). If $t \in(0, \infty)$, then the symbol $H^{t}(\mathrm{D})$ will be used to denote the Hardy class of all holomorphic functions $g: \mathbb{D} \rightarrow \mathbb{C}$ which fulfill

$$
\sup _{r \in[0,1)} \frac{1}{2 \pi} \int_{\mathbf{T}}|g(r z)|^{t} \tilde{\lambda}(d z)<+\infty
$$


whereas $H^{\infty}(\mathbb{D})$ designates the set of all holomorphic and bounded functions $g: \mathbb{D} \rightarrow \mathbb{C}$. Observe that

$$
H^{s}(\mathbb{D}) \subset H^{t}(\mathbb{D}) \subset \mathcal{N}_{+}(\mathbb{D}) \subset \mathcal{N}(\mathbb{D}) \subset \mathcal{N} \mathcal{M}(\mathbb{D})
$$

where $0<t<s \leqq \infty$.

In our following considerations, the next theorem (see, e.g., [10, Theorem 2.11]) will play a key role. It can be conceived as a maximum modulus principle for the Smirnov class. Hereby and in the following, let $\mathfrak{B}$ be the $\sigma$-algebra of all Borel subsets of $\mathbf{T}$.

Theorem 1: Let $p \in(0, \infty]$, and let $f \in \mathcal{N}_{+}(\mathrm{DD})$ be such that $\underline{f}$ belongs to $\mathcal{L}^{p}(\mathbf{T}, \mathfrak{B}, \bar{\lambda} / 2 \pi ; \mathbb{C})$. Then $f$ belongs to $H^{p}(\mathbb{D})$.

For the convenience of the reader, now we are going to recall some facts on outer functions which belong to the matricial Smirnov class. A function $\Phi \in\left[\mathcal{N}_{+}(\mathbb{D})\right]^{m \times m}$ is called outer (in $\left[\mathcal{N}_{+}(\mathbb{D})\right]^{m \times m}$ ) if $\operatorname{det} \Phi$ is outer in $\mathcal{N}(\mathbb{D})$. An outer function $\Phi \in$ $\left[\mathcal{N}_{+}(\mathbb{D})\right]^{m \times m}$ is called normalized if $\Phi(0)$ is nonnegative Hermitian. The following useful properties of matrix-valued outer functions can be taken, e.g., from AROV [2].

Remark 1: $\Phi \in\left[\mathcal{N}_{+}(\mathrm{DD})\right]^{m \times m}$ is outer if and only if $\Phi$ admits the representation $\Phi=\frac{1}{\varphi_{2}} \Phi_{1}$ with some outer functions $\Phi_{1} \in\left[H^{\infty}(\mathrm{ID})\right]^{m \times m}$ and $\varphi_{2} \in H^{\infty}(\mathbb{D})$.

Remark 2: (a) If $\Phi$ is an outer function in $\left[\mathcal{N}_{+}(\mathbb{D})\right]^{m \times m}$, then $\operatorname{det} \Phi(z) \neq 0$ for all $z \in \mathbb{D}$, and $\Phi^{-1}$ is an outer function in $\left[\mathcal{N}_{+}(\mathbb{D})\right]^{m \times m}$.

(b) If $\Phi \in\left[\mathcal{N}_{+}(\mathbb{D})\right]^{m \times m}$ satisfies det $\Phi(z) \neq 0$ for all $z \in \mathbb{D}$ and if $\Phi^{-1}$ belongs to $\left[\mathcal{N}_{+}(\mathbb{D})\right]^{m \times m}$, then both $\Phi$ and $\Phi^{-1}$ are outer functions in $\left[\mathcal{N}_{+}(\mathbb{D})\right]^{m \times m}$.

Remark 3: If both $\Phi$ and $\Psi$ are outer functions in $\left[\mathcal{N}_{+}(\mathrm{DD})\right]^{m \times m}$, then the product $\Phi \Psi$ is also an outer function in $\left[\mathcal{N}_{+}(\mathbb{D})\right]^{m \times m}$.

Remark 4: $\Phi \in\left[H^{2}(\mathrm{DD})\right]^{m \times m}$ is outer if and only if $\operatorname{det} \Phi$ is outer in $H^{2 / m}(\mathrm{D})$.

Remark 5: Let $\varrho \in(1, \infty)$, and let $\Phi$ be an $m \times m$ matrix-valued function which is holomorphic in $K(0 ; \varrho):=\{z \in \mathbb{C}:|z|<\varrho\}$ and which satisfies $\operatorname{det} \Phi(z) \neq 0$ for all $z \in K(0 ; \varrho)$. Then the restriction $R s t r . \mathbb{D} \Phi$ of $\Phi$ on'to $\mathbb{D}$ is an outer function in $\left[H^{\infty}(\mathbb{D})\right]^{m \times m}$.

A function $\Phi \in\left[H^{2}(\mathbb{D})\right]^{m \times m}$ is called left maximal if, for each $\Sigma \in\left[H^{2}(\mathbb{D})\right]^{m \times m}$ with $\left\langle\underline{\Sigma} \underline{\Sigma}^{*}\right\rangle=\left\langle\underline{\Phi} \underline{\Phi}^{*}\right\rangle$, the inequality $\Sigma(0) \Sigma^{*}(0) \leqq \Phi(0) \Phi^{*}(0)$ holds true. Analogously, a function $\Psi \in\left[H^{2}(\mathbb{D})\right]^{m \times m}$ is said to be right maximal if, for each $\Xi \in\left[H^{2}(\mathbb{D})\right]^{m \times m}$ with $\langle\Xi \Xi \Xi\rangle=\left\langle\underline{\Psi}^{*} \underline{\Psi}\right\rangle$, the inequality $\Xi^{*}(0) \Xi(0) \leqq \Psi^{*}(0) \Psi(0)$ holds true. A left (respectively, right) maximal function is called normalized if it has a nonnegative Hermitian value at $z=0$. Every left maximal function $\Phi$ and every right maximal function $\Psi$ in $\left[H^{2}(\mathbb{D})\right]^{m \times m}$ satisfy $\operatorname{rank} \Phi(z)=\operatorname{rank} \Phi(0)$ and $\operatorname{rank} \Psi(z)=\operatorname{rank} \Psi(0)$ for all $z \in \mathbb{D}$ (see, e.g., MASANI $[20])$.

Lemma 2 (see, e.g., MASANI [20]): Let $\Phi \in\left[H^{2}(\mathbb{D})\right]^{m \times m}$. Then the following three statements are equivalent:

(i) $\Phi$ is left maximal and $\operatorname{rank} \Phi(0)=m$. 
(ii) $\Phi$ is right maximal and $\operatorname{rank} \Phi(0)=m$.

(iii) $\Phi$ is outer.

Let $\mathbb{C}_{\geq}^{m \times m}$ be the set of all nonnegative Hermitian $m \times m$ matrices, and let $W: \mathbf{T} \rightarrow \mathbb{C}_{\geq}^{m \times m}$ be Lebesgue integrable. A function $\Sigma \in\left[H^{2}(\mathbb{D})\right]^{m \times m}$ is called a left (respectively, right) minorant of $\langle W\rangle$ if

$$
\underline{\Sigma} \underline{\Sigma}^{*} \leqq W \quad \tilde{\lambda} \text {-a.e. on } \mathbf{T} \quad \text { (respectively, } \underline{\Sigma}^{*} \underline{\Sigma} \leqq W \quad \tilde{\lambda} \text {-a.e. on } \mathbf{T} \text { ) . }
$$

A function $\Sigma \in\left[H^{2}(\mathbb{D})\right]^{m \times m}$ is said to be a largest left minorant (respectively, a largest right minorant) of $\langle W\rangle$ if the following two conditions are satisfied:

(i) Inequality (3) is fulfilled.

(ii) If $\Xi$ is an arbitrary left (respectively, right) minorant of $\langle W\rangle$, then $\Xi(0) \Xi *(0) \leqq$ $\Sigma(0) \Sigma^{*}(0)$ (respectively, $\Xi^{*}(0) \Xi(0) \leqq \Sigma^{*}(0) \Sigma(0)$ ).

A function $\Sigma \in\left[H^{2}(\mathbb{D})\right]^{m \times m}$ is called a left (respectively, right) spectral factor of $\langle W\rangle$ if $\underline{\Sigma} \underline{\Sigma}^{*}=W \cdot \tilde{\lambda}$ - a.e. on $\mathbf{T}$ (respectively, $\underline{\Sigma}^{*} \underline{\Sigma}=W \tilde{\lambda}$ - a.e. on $\mathbf{T}$ ).

The following theorem, which shows the existence of largest minorants, was proved by MASANI [19] in the context of prediction theory. Later SZ.-NAGY and FOIAS [25] developed an operatorial version.

Theorem 2: Let $W: \mathbf{T} \rightarrow \mathbb{C}_{\geq}^{m \times m}$ be Lebesgue integrable. Then:

(a) There exists a unique largest normalized left minorant $\Phi_{0}$ of $\langle W\rangle$. This function $\Phi_{0}$ is left maximal.

(b) If $\Phi$ is a largest left minorant of $\langle W\rangle$, then $\Phi=\Phi_{0} U$. with some unitary matrix $U$. In particular, $\Phi$ is left maximal.

(c) There exists a unique largest normalized right minorant $\Psi_{0}$ of $\langle W\rangle$. This function $\Psi_{0}$ is right maximal.

(d) If $\Psi$ is a largest right minorant of $\langle W\rangle$, then $\Psi=V \Psi_{0}$ with some unitary matrix $V$. In particular, $\Psi$ is right maximal.

(e) If $\int_{\mathbf{T}} \log (\operatorname{det} W) d \tilde{\lambda}>-\infty$, then $\Phi_{0}$ and $\Psi_{0}$ are an outer left spectral factor of $\langle W\rangle$ and an outer right spectral factor of $\langle W\rangle$, respectively.

\section{Some Interrelations between Matrix-valued Functions Belonging to the Classes of Schur and Carathéodory}

An important subclass of $\left[H^{\infty}(\mathbb{D})\right]^{p \times q}$ is the so-called Schur class $\mathcal{S}_{p \times q}(\mathbb{D})$. It consists of all $p \times q$ matrix-valued functions defined on $\mathbb{D}$ which are both holomorphic and contractive in ID. If we speak of a $p \times q$ Schur function $f$, then we mean that $f$ belongs to $\mathcal{S}_{p \times q}(\mathrm{ID})$.

Lemma 3: Let $f \in \mathcal{S}_{m \times m}(\mathbb{D})$. 
(a) There are at most $m$ numbers $\eta \in \mathbf{T}$ such that $\operatorname{det}(\eta I+f)$ has a zero in' $\mathbb{D}$.

(b) If $\eta \in \mathbf{T}$ is such that $\operatorname{det}\left[\eta I+f\left(z_{0}\right)\right]=0$ for some $z_{0} \in \mathbb{D}$, then $\operatorname{det}(\eta I+f)$ identically vanishes in $\mathrm{D}$.

(c) If $\eta \in \mathbf{T}$ is such that $\operatorname{det}\left[\eta I+f\left(z_{0}\right)\right] \neq 0$ for some $z_{0} \in \mathbb{D}$, then $\operatorname{det}(\eta I+f)$ nowhere vanishes in $\mathrm{D}$.

Proof: Apply Lemma 2.1.6 in [8]

A function $\Omega: \mathbb{D} \rightarrow \mathbb{C}^{m \times m}$ is called $m \times m$ Carathéodory function if $\Omega$ is holomorphic and has nonnegative Hermitian real part $\operatorname{Re} \Omega(z)$ for all $z \in \mathbb{D}$. We will write $\mathcal{C}_{m}(\mathbb{D})$ for the set of all $m \times m$ Carathéodory functions. There are several interesting interrelations between matricial Schur functions and matricial Carathéodory functions. It will be useful to recall some of them.

Proposition 1: The following statements hold true:

(a) Let $\Omega$ belong to $\mathcal{C}_{m}(\mathbb{D})$. Then $\operatorname{det}(I+\Omega)$ does not vanish in $\mathbb{D}$. The function $f:=(I-\Omega)(I+\Omega)^{-1}$ belongs to $\mathcal{C}_{m}(\mathrm{ID})$ and fulfills

$$
I+f=2(I+\Omega)^{-1} \text {. }
$$

In particular, det $(I+f)$ has no zeros in $\mathrm{DD}$. Moreover,

$$
\Omega=(I-f)(I+f)^{-1}=(I+f)^{-1}(I-f)
$$

and

$$
\operatorname{rank}[\operatorname{Re} \Omega(z)]=\operatorname{rank}\left[I-f^{*}(z) f(z)\right]=\operatorname{rank}\left[I-f^{*}(0) f(0)\right]
$$

for all $z \in \mathbb{D}$.

(b) Let $f \in \mathcal{S}_{m \times m}(\mathbb{D})$, and let $\eta \in \mathbb{E}$ be such that $\operatorname{det}\left[\eta I+f\left(z_{0}\right)\right] \neq 0$ for some $z_{0} \in \mathbb{D}$. Then $\Omega:=(\eta I-f)(\eta I+f)^{-1}$ belongs to $\mathcal{C}_{m}(\mathbb{D})$. Furthermore,

$$
f=\eta(I-\Omega)(I+\Omega)^{-1}=\eta(I+\Omega)^{-1}(I-\Omega) .
$$

A proof of Proposition 1 can be found in [8, Propositions 2.1.2, 2.1.3 and part (f) of Lemma 1.3.12].

The following result, which is taken from AROV [2], provides an interesting connection between Schur functions and outer functions.

Proposition 2: Let $f \in \mathcal{S}_{m \times m}$ be such that $\operatorname{det}(I+f)$ does not identically vanish. Then $I+f$ is an outer function in $\left[H^{\infty}(\mathbb{D})\right]^{m \times m}$.

Corollary 1: Let $f \in \mathcal{S}_{m \times m}$ be such that $\operatorname{det}(I+f)$ does not identically vanish. Then $\frac{1}{2}(I+f)$ is an outer function in $\mathcal{S}_{m \times m}(\mathbb{D})$.

Proof: Use Proposition 2, Remarks 3 and 5 and the inequality

$$
\left\|\frac{1}{2}(I+f)\right\| \leqq \frac{1}{2}(\|I\|+\|f\|) \leqq 1 .
$$


Corollary 2: $\mathcal{C}_{m}(\mathbb{D}) \subseteq\left[\mathcal{N}_{+}(\mathbb{D})\right]^{m \times m}$

Proof: Let $\Omega \in \mathcal{C}_{m}(\mathbb{D})$, and let $f:=(I-\Omega)(I+\Omega)^{-1}$. According to part (a) of Proposition $l$ and Proposition 2, $I+f$ is an outer function in $\left[H^{\infty}(\mathbb{D})\right]^{m \times m}$. Part (b) of Remark 2 yields that $(I+f)^{-1} \in\left[\mathcal{N}_{+}(\mathbb{D})\right]^{m \times m}$. Since $I-f$ obviously belongs to $\left[H^{\infty}(\mathbb{D})\right]^{m \times m}$, the fact that $\mathcal{N}_{+}(\mathbb{D})$ is a subalgebra of $\mathcal{N}(\mathbb{D})$ implies that $(I-f)(I+f)^{-1}$ belongs to $\left[\mathcal{N}_{+}(\mathbb{D})\right]^{m \times m}$. By virtue of part (a) of Proposition 1 , we have the identity $\Omega=(I-f)(I+f)^{-1}$. Thus, $\Omega \in\left[\mathcal{N}_{+}(\mathbb{D})\right]^{m \times m}$

Observe that, as a consequence of Corollary 2, every $m \times m$ Carathéodory function has boundary values $\underline{\Omega} \dot{\lambda}$-a.e. on $\mathbf{T}$.

Proposition 3: Let $\Omega \in \mathcal{C}_{m}(\mathbb{D})$. Then: ,

(a) $(I+\Omega)^{-1}$ is an outer function in $\mathcal{S}_{m \times m}(\mathbb{D})$.

(b) $f:=(I-\Omega)(I+\Omega)^{-1}$ is an outer function in $\left[H^{\infty}(\mathbb{D})\right]^{m \times m}$ if and only if $I-\Omega$ is an outer function in $\left[\mathcal{N}_{+}(\mathbb{D})\right]^{m \times m}$.

Proof: (a) Using properties of the Cayley transformation (see, e.g., Lemma 1.3.12 in [8]) we obtain that $f$ is an $m \times m$ Schur function which fulfills $(I+\Omega)^{-1}=\frac{1}{2}(I+f)$. In particular, $\operatorname{det}(I+f)$ does not identically vanish. Since $f$ belongs to $\mathcal{S}_{m \times m}(\mathbb{D D})$, Corollary 1 shows that $(I+\Omega)^{-1}$ is an outer function in $\mathcal{S}_{m \times m}(\mathrm{D})$.

(b) From part (a) and Remark 2 we see that $I+\Omega$ is an outer function in $\left[\mathcal{N}_{+}(\mathbb{D})\right]^{m \times m}$. Remark 3 yields the asserted equivalence

Proposition 4: Let $\Omega \in \mathcal{C}_{m}(\mathbb{D})$, and let $f:=(I-\Omega)(I+\Omega)^{-1}$. Then the following statements are equivalent:

(i) $\Omega$ is an outer function in $\left[\mathcal{N}_{+}(\mathrm{ID})\right]^{m \times m}$.

(ii) $\operatorname{det} \Omega$ does not identically vanish in $\mathbb{D}$.

(iii) $\operatorname{det}(I-f)$ does not identically vanish in $\mathbb{D}$.

(iv) $I-f$ is an outer function in $\left[H^{\infty}(\mathbb{D})\right]^{m \times m}$.

Proof: Part (b) of Proposition 1 shows that $f$ is an $m \times m$ Schur function for which $I+f$ does not vanish in $\mathbb{D}$. Thus, we see the equivalences as follows:

'(i) $\Rightarrow$ (ii)' : Use Remark 2.

'(ii) $\Rightarrow$ (iii)' : Apply part (a) of Proposition 1.

'(iii) $\Rightarrow$ (iv)' : The function $-f$ is obviously an $m \times m$ Schur function.

Hence, condition (iii) and Proposition 2 imply (iv).

'(iv) $\Rightarrow$ (i)' : Proposition 2 and Remark 2 yicld that $I+f$ and $(I+f)^{-1}$ arc outer functions in $\left[\mathcal{N}_{+}(\mathrm{DD})\right]^{m \times m}$. Therefore, we get from part (a) of Proposition 1 and Remark 3 that (i) holds true

Proposition 5: Let $\Omega \in \mathcal{C}_{m}(\mathrm{D})$ be such that $\operatorname{det} \Omega$ does not identically vanish. Then:

(a) The function det $\dot{\Omega}$ does not vanish in $\mathbb{D}$, and $\Omega^{-1}$ belongs to $\mathcal{C}_{m}(\mathbb{D})$. 
(b) $\Omega^{-1}$ is an outer function in $\left[\mathcal{N}_{+}(\mathbb{D})\right]^{m \times m}$.

(c) $\operatorname{Re} \underline{\Omega^{-1}}=\underline{\Omega^{-1}} \cdot \operatorname{Re} \underline{\Omega} \cdot\left(\underline{\Omega^{-1}}\right)^{*}=\left(\underline{\Omega^{-1}}\right)^{*} \cdot \operatorname{Re} \underline{\Omega} \cdot \underline{\Omega^{-1}} \tilde{\lambda}$-a.e. on $\mathrm{T}$.

Proof: By virtue of Proposition 4 and Remark 2, det $\Omega$ nowhere vanishes in $\mathbb{D}$. Thus, $\Omega^{-1}$. belongs to $\mathcal{C}_{m}$ (ID) (see, e.g., [8, Lemma 2.1.10]). To verify part (b) it remains to apply part (a), Corollary 2 and part (b) of Remark 2. The identities stated in (c) are obvious

\section{On Connections between the Largest Minorants Associated with a Cayley-linked Pair Consisting of a Carathéodory Function and a Schur Function}

First we will turn our attention to crossconnections between matrix-valued Carathéodory functions and nonnegative Hermitian-valued Borel measures on the unit circle.

Remark 6: Let $\Omega$ be an $m \times m$ Carathéodory function. Then the matricial version of the famous classical theorem of F. Riesz and Herglotz (see, e.g., [8, Theorem 2.2.2]) shows that there is a unique $m \times m$ nonnegative Hermitian-valued Borel measure on the unit circle $\mathbf{T}$ such that

$$
\Omega(w)=\int_{\mathbf{T}} \frac{z+w}{z-w} F(d z)+i \operatorname{Im} \Omega(0)
$$

for all $w \in \mathbb{D}$. This measure $F$ is called the $F$. Riesz-Herglotz measure associated with the matrix-valued Carathéodory function $\Omega$. If

$$
\Omega(z)=\sum_{k=0}^{\infty} \Gamma_{k} z^{k}, \quad z \in \mathbb{D},
$$

is the Taylor series representation of $\Omega$, then the Fourier coefficients

$$
C_{k}^{(F)}:=\int_{\mathbf{T}} z^{-k} F(d z), \quad k \in \mathbb{N}_{0},
$$

of $F$ satisfy

$$
C_{0}^{(F)}=\operatorname{Re} \Gamma_{0} \text { and } C_{k+1}^{(F)}=\frac{1}{2} \Gamma_{k+1}, \quad k \in \mathbb{N}_{0}
$$

Lemma 4: Let $\Omega \in \mathcal{C}_{m}(\mathrm{DD})$, and let $F$ be the $F$. Riesz-Herglotz measure associated with $\Omega$. Then $\operatorname{Re} \underline{\Omega}$ is the Radon-Nikodym dcrivative of the absolutely continuous part $F_{a}$ of $F$ in the Lebesgue decomposition of $F$ with respect to the normalized linear Lebesgue-Borel measure $\tilde{\lambda} / 2 \pi$ on $\mathbb{T}$. In particular,

$$
\operatorname{Re} \underline{\Omega} \in \mathcal{L}^{1}(\mathbf{T}, \mathfrak{B}, \tilde{\lambda} / 2 \pi ; \mathbb{C}) .
$$


Proof: For $z \in \mathbf{T}$ and $r \in[0,1)$, we get from (7) that

$$
\operatorname{Re}[\Omega(r z)]=\int_{\mathbf{T}} \operatorname{Re}\left(\frac{\xi+r z}{\xi-r z}\right) F(d \xi)=\int_{\mathbf{T}} \frac{1-r^{2}}{|\xi z-r|^{2}} F(d \xi) .
$$

Let $W$ be the Radon-Nikodym derivative of $F_{a}$ with respect to $\tilde{\lambda} / 2 \pi$. From [10, Theorem 1.2] and (12) we see thus that

$$
W(z)=\lim _{r \rightarrow 1-0} \int_{\mathbf{T}} \frac{1-r^{2}}{|\xi z-r|^{2}} F(d \xi)=\lim _{r \rightarrow 1-0} \operatorname{Re}[\Omega(r z)]=\underline{\Omega}(z)
$$

holds true for $\tilde{\lambda}$-almost all $z \in \mathbb{T}$. The proof is finished

The next theorem will prove to be essential for our following considerations.

Theorem 3: Let $\Omega \in \mathcal{C}_{m}(\mathbb{D})$, and $f:=(I-\Omega)(I+\Omega)^{-1}$.

(a) Let $\Phi$ be a largest left minorant of $\langle\operatorname{Re} \underline{\Omega}\rangle$. Then the $m \times m$ Schur function $\varphi:=$ $2(I+\Omega)^{-1} \Phi$ is a largest left minorant of $\left\langle I-\underline{f}^{*}\right\rangle$. If $\Omega(0)=I$, then $\Phi(0)=\varphi(0)$.

(b) Let $\varphi$ be an arbitrary largest left minorant of $\left(I-f f^{*}\right\rangle$. Then $\Phi:=(I+f)^{-1} \varphi$ is a largest left minorant of $\langle\operatorname{Re} \underline{\Omega}\rangle$.

(c) Let $\Psi$ be a largest right minorant of $\langle\operatorname{Re} \underline{\Omega}\rangle$. Then the $m \times m$ Schur function $\psi:=$ $2 \Psi(I+\Omega)^{-1}$ is a largest right minorant of $\left\langle I-\underline{f} \underline{f}^{*}\right\rangle$. If $\Omega(0)=I$, then $\Psi(0)=\psi(0)$.

(d) Let $\psi$ be an arbitrary largest right minorant of $\left\langle I-\underline{f} \underline{f}^{*}\right\rangle$. Then $\Psi:=\psi(I+f)^{-1}$ is a largest right minorant of $\langle\operatorname{Re} \underline{\Omega}\rangle$.

Proof: By virtue of Proposition 1, the $m \times m$ Schur function $f$ satisfies (4) and (5). Moreover, we see that $\operatorname{det}(I+f)$ does not vanish in $\mathrm{DD}$. Proposition 2 yields that $I+f$ is an outer function in $\left[H^{\infty}(\mathrm{ID})\right]^{m \times m}$. Hence, Lemma 2 implies that $I+f$ is left maximal. Moreover, part (a) of Remark 2 shows

$$
(I+f)^{-1} \in\left[\mathcal{N}_{+}(\mathbb{D})\right]^{m \times m} .
$$

From Lemma 4 we see that (11) holds true.

(a) In view of (4), we see $\varphi=(I+f) \Phi$. Thus, as a product of functions which belong to $\left[H^{\infty}(\mathrm{DD})\right]^{m \times m}$ and $\left[H^{2}(\mathbb{D})\right]^{m \times m}$, respectively the function $\varphi$ also belongs to $\left[H^{2}(\mathbb{D})\right]^{m \times m}$. Since $\Phi$ is a left minorant of $\operatorname{Re} \underline{\Omega}$, we have $\underline{\Phi} \underline{\Phi}^{*} \leqq \operatorname{Re} \underline{\Omega} \bar{\lambda}$-a.e. on $\mathbf{T}$. Thus, .

$$
\begin{aligned}
\underline{\varphi} \underline{\varphi}^{*} & =4(I+\underline{\Omega})^{-1} \underline{\Phi} \underline{\Phi}^{*}\left[(I+\underline{\Omega})^{-1}\right]^{*} \\
& \leqq 4(I+\underline{\Omega})^{-1} \cdot \operatorname{Re} \underline{\Omega} \cdot\left[(I+\underline{\Omega})^{-1}\right]^{*} \tilde{\lambda} \text {-a.e. on } \mathbf{T} .
\end{aligned}
$$

Using properties of the Cayley transformation (see [8, part (i) of Lemma 1.3.12]), it follows $\underline{\varphi} \underline{\varphi}^{*} \leqq I-\underline{f} \underline{f}^{*} \quad \bar{\lambda}$-a.e. on $\mathbb{T}$. Thus, $\varphi$ is a left minorant of $\left\langle I-f \underline{f} \underline{f}^{*}\right\rangle$. Now let $\eta$ be an arbitrary left minorant of $\left\langle I-\underline{f} \underline{f}^{*}\right\rangle$. Then $\eta$ belongs to $\left[H^{2}(\mathbb{D})\right]^{\frac{f}{m} \frac{f}{x m}} \subseteq\left[\mathcal{N}_{+}(\mathbb{D})\right]^{m \times m}$ and satisfies

$$
\underline{\eta} \underline{\eta}^{*} \leqq I-\underline{f} \underline{f}^{*} \leqq I \quad \tilde{\lambda} \text {-a.e. on } \mathbf{T} \text {. }
$$


Theorem 1 yields then $\eta \in \mathcal{S}_{m \times m}(\mathbb{D})$. As a product of two functions which belong to the Smirnov class, the function $\Sigma:=(I+f)^{-1} \eta$ also belongs to $\left[\mathcal{N}_{+}(\mathbb{D})\right]^{m \times m}$. Using properties of the Cayley transform (see part $(\mathrm{g})$ of Theorem 1.3.12 in [8]), we get

$$
\begin{aligned}
\underline{\Sigma} \underline{\Sigma} & =(I+\underline{f})^{-1} \underline{\eta} \underline{\eta}^{*}\left[(I+\underline{f})^{-1}\right]^{*} \\
& \leq(I+\underline{f})^{-1}\left(I+\underline{f} \underline{f}^{*}\right)^{-1}\left[(I+\underline{f})^{-1}\right]^{*}=\operatorname{Re} \underline{\Omega} \quad \tilde{\lambda} \text {-a.e. on } \mathbf{T} .
\end{aligned}
$$

From (11) and Lemma 1 we see then $\underline{\Sigma} \in\left[\mathcal{L}^{2}(\mathbf{T}, \mathfrak{B}, \bar{\lambda} / 2 \pi ; \mathbb{C})\right]^{m \times m}$. Thus, Theorem 1 provides that $\Sigma$ belongs to the Hardy space $\left[H^{2}(\mathbb{D})\right]^{m \times m}$. Since $\Phi$ is a largest left minorant of $\langle\operatorname{Re} \underline{\Omega}\rangle$, we obtain from (14) that $\Sigma(0) \Sigma^{*}(0) \leqq \Phi(0) \Phi^{-}(0)$ holds true. Then it follows

$$
\begin{aligned}
\eta(0) \eta^{*}(0) & =[I+f(0)] \Sigma(0) \Sigma^{*}(0)[I+f(0)]^{*} \\
& =[I+f(0)] \Phi(0) \Phi^{*}(0)[I+f(0)]^{*}=\varphi(0) \varphi^{*}(0) .
\end{aligned}
$$

Therefore, $\varphi$ is a largest left minorant of $\left\langle I-\underline{f} \underline{f}^{*}\right\rangle$. If $\Omega(0)=I$, then $\varphi(0)=$ $2[I+\Omega(0)]^{-1} \Phi(0)=\Phi(0)$.

(b) By assumption $\varphi$ belongs to $\left[H^{2}(\mathbb{D})\right]^{m \times m}$ and satisfies

$$
\underline{\varphi} \underline{\varphi}^{*} \leqq I-\underline{f} \underline{f}^{*} \quad \tilde{\lambda} \text {-a.e. on } \mathbb{T} \text {. }
$$

Moreover, if $\eta \in\left[H^{2}(\mathrm{DD})\right]^{m \times m}$ is an arbitrary left minorant of $\left(I-\underline{f} \underline{f}^{*}\right)$, then

$$
\eta(0) \eta^{*}(0) \leqq \varphi(0) \varphi^{*}(0)
$$

In view of (13), the function $\Phi$ is a product of functions which belong to the Smirnov class. Hence, $\Phi$ belongs to $\left[\mathcal{N}_{+}(\mathbb{D})\right]^{m \times m}$. Using Lemma 1.3 .12 in [8] and (15), we get

$$
\begin{aligned}
\underline{\Phi}^{*} & =(I+\underline{f})^{-1} \underline{\varphi} \underline{\varphi}^{*}\left[(I+\underline{f})^{-1}\right]^{*} \\
& =(I+\underline{f})^{-1}\left(I-\underline{f} \underline{f}^{*}\right)\left[(I+\underline{f})^{-1}\right]^{*}=\operatorname{Re} \underline{\Omega} \quad \dot{\lambda} \text {-a.e. on } \mathbf{T} .
\end{aligned}
$$

From (11) and Lemma 1 , then we can conclude that $\Phi$ belongs to $\left[\mathcal{L}^{2}(\mathbb{T}, \mathfrak{B}, \bar{\lambda} / 2 \pi ; \mathbb{C})\right]^{m \times m}$. Theorem 1 thus implies $\Phi \in\left[H^{2}(\mathbb{D})\right]^{\mathrm{m} \times \mathrm{m}}$. Therefore, $\Phi$ is a left minorant of $(\operatorname{Re} \Omega\rangle$. Now let $\Sigma \in\left[H^{2}(\mathbb{D})\right]^{m \times m}$ be an arbitrary left minorant of $\langle\operatorname{Re} \underline{\Omega}\rangle$. Then $\eta:=(I+f) \Sigma$ belongs to $\left[H^{2}(\mathbb{D})\right]^{m \times m}$ and satisfies

$$
\underline{\eta} \underline{\eta}^{*}=(I+\underline{f}) \underline{\Sigma} \underline{\Sigma}(I+\underline{f})^{*} \leqq(I+\underline{f}) \cdot \operatorname{Re} \underline{\Omega} \cdot(I+\underline{f})^{*} \quad \dot{\lambda}-\text { a.e. on } \mathbf{T} .
$$

The last identity in (17) thus yields $\underline{\eta} \underline{\eta}^{*} \leqq I-\underline{f} \underline{f}^{*} \bar{\lambda}$-a.e. on $\mathbf{T}$, i.e. $\eta$ is a left minorant of $\left\langle I-f f^{*}\right\rangle$. Since $\varphi$ is a largest left minorant of $\left\langle I-\underline{f} \underline{f}^{*}\right\rangle$, then we obtain $\eta(0) \eta^{*}(0) \leqq$ $\varphi(0) \varphi \cdot(0)$. Propósition 1 shows $\operatorname{det}[I+f(0)] \neq 0$. Thus it follows

$$
\begin{aligned}
\Sigma(0) \Sigma^{*}(0) & =[I+f(0)]^{-1} \eta(0) \eta^{*}(0)\left([I+f(0)]^{-1}\right)^{\bullet} \\
& \leqq[I+f(0)]^{-1} \varphi(0) \varphi^{*}(0)\left([I+f(0)]^{-1}\right)^{*}=\Phi(0) \Phi^{*}(0) .
\end{aligned}
$$

This completes the proof of part (b). Parts (c) and (d) can be analogously verified 
In the operator case, the paper [5] of CONSTANTINECSU contains investigations on largest minorants associated with a semispectral measure $F$. In particular, using appropriate operator functions taken from the Naimark dilation of $F$, Theorem 4.1 and Corollary 4.4 in [5] describe some distinguished interrelations between the largest minorants associated with expressions comparable with $\langle\operatorname{Re} \underline{\Omega}\rangle$ and $\left\langle I-\underline{f} \underline{f}^{*}\right\rangle$. Now we will establish relations between the largest minorants associated with a Carathéodory function $\Omega$ and its inverse $\Omega^{-1}$, respectively.

Theorem 4: Let $\Omega \in \mathcal{C}_{m}$ (ID) be such that $\operatorname{det} \Omega$ does not identically vanish.

(a) Let $\Phi$ be a largest left minorant of $\langle\operatorname{Re} \underline{\Omega}\rangle$. Then $\Phi_{1}:=\Omega^{-1} \Phi$ is a largest left minorant of $\left\langle\operatorname{Re} \underline{\Omega^{-1}}\right\rangle$. If $\Omega(0)=I$, then $\Phi_{1}(0)=\Phi(0)$.

(b) Let $\Psi$ be a largest right minorant of $(\operatorname{Re} \underline{\Omega}\rangle$. Then $\Psi_{1}:=\Psi \Omega^{-1}$. is a largest right minorant of $\left\langle\operatorname{Re} \underline{\Omega^{-1}}\right\rangle$. If $\Omega(0)=I$, then $\Psi_{1}(0)=\Psi(0)$.

Proof: By virtue of Proposition $4, \Omega$ is an outer function in $\left[\mathcal{N}_{+}(\mathbb{D})\right]^{m \times m}$. Remark 2 shows then that $\Omega^{-1}$ is an outer function in $\left[\mathcal{N}_{+}(\mathbb{D})\right]^{m \times m}$. Since $\Phi \in\left[H^{2}(\mathbb{D})\right]^{m \times m} \subseteq$ $\left[\mathcal{N}_{+}(\mathbb{D})\right]^{m \times m}$, the function $\Phi_{1}$, as a product of functions which belong to the Smirnov class, is a member of $\left[\mathcal{N}_{+}(\mathrm{D})\right]^{m \times m}$. Since $\Phi$ is a largest left minorant of $\langle\operatorname{Re} \underline{\Omega}\rangle$, part (b) of Proposition 5 yields

$$
\underline{\Phi_{1}} \underline{\Phi}^{*}=\underline{\Omega^{-1}} \underline{\Phi} \underline{\Phi}\left(\underline{\Omega^{-1}}\right)^{*} \leqq \underline{\Omega^{-1}} \cdot \operatorname{Re} \underline{\Omega} \cdot\left(\underline{\Omega^{-1}}\right)^{*}=\operatorname{Re} \underline{\Omega^{-1}} \tilde{\lambda} \text {-a.e. on } \bar{T} .
$$

Part (a) of Proposition 5 shows that $\Omega^{-1}$ belongs to $\mathcal{C}_{m}(\mathbb{D})$. Hence, Lemma 4 provides $\operatorname{Re} \underline{\Omega^{-1}} \in \mathcal{L}^{1}(\mathbb{T}, \mathfrak{B}, \tilde{\lambda} / 2 \pi ; \mathbb{C})$. Thus, Lemma 1 and inequality $(19)$ imply that $\Phi_{1} \in \mathcal{L}^{2}(\mathbb{T}, \mathfrak{B}, \tilde{\lambda} / 2 \pi ; \mathbb{C})$. From Theorem 1 we then obtain that $\Phi_{1}$ belongs to $\left[H^{2}(\mathbb{D})\right]^{m \times m}$. Therefore, $\Phi_{1}$ is a left minorant of $\left\langle\operatorname{Re} \Omega^{-1}\right\rangle$. Now let $\Sigma_{1}$ be an arbitrary left minorant of $\left\langle\operatorname{Re} \Omega^{-1}\right\rangle$. Because of Corollary 2, the $m \times m$ Carathéodory function $\Omega$ belongs to $\left[\mathcal{N}_{+}(\mathbb{D})\right]^{m \times m}$. Since $\Sigma_{1} \in\left[H^{2}(\mathbb{D})\right]^{m \times m}$, we obtain that $\Sigma:=\Omega \Sigma_{1}$ belongs to $\left[\mathcal{N}_{+}(\mathbb{D})\right]^{m \times m}$. Consequently, it follows from part (b) of Proposition 5 that

$$
\underline{\Sigma} \underline{\Sigma}^{*}=\underline{\Omega} \underline{\Sigma_{1}} \underline{\Sigma_{1}} \underline{\Omega}^{*} \leq \underline{\Omega} \cdot \operatorname{Re} \underline{\Omega^{-1}} \cdot \underline{\Omega}^{*}=\operatorname{Re} \underline{\Omega} \quad \tilde{\lambda} \text {-a.e. on } \mathrm{T} .
$$

By virtue of Lemma 4 , Re $\underline{\Omega}$ belongs to $\mathcal{L}^{1}(\mathbf{T}, \mathfrak{B}, \tilde{\lambda} / 2 \pi ; \mathbb{C})$. Thus, Lemma 1 and inequality (20) show that $\underline{\Sigma}$ belongs to $\mathcal{L}^{2}(\mathbf{T}, \mathfrak{B}, \tilde{\lambda} / 2 \pi ; \mathbb{C})$. Then we see from Theorem 1 that $\Sigma$ belongs to $\left[H^{2}(\mathbb{D})\right]^{m \times m}$, i.e. $\Sigma$ is a left minorant of $\langle\operatorname{Re} \underline{\Omega}\rangle$. Since $\Phi$ is a largest left minorant of $\langle\operatorname{Re} \underline{\Omega}\rangle$, we get $\Sigma(0) \Sigma^{*}(0) \leqq \Phi(0) \Phi^{*}(0)$ and, therefore,

$$
\begin{aligned}
\Sigma_{1}(0) \Sigma_{1}^{*}(0) & =[\Omega(0)]^{-1} \Sigma(0) \Sigma^{*}(0)\left([\Omega(0)]^{-1}\right)^{*} \\
& =[\Omega(0)]^{-1} \Phi(0) \Phi^{*}(0)\left([\Omega(0)]^{-1}\right)^{*}=\Phi_{1}(0) \Phi_{1}^{*}(0)
\end{aligned}
$$

Consequently, $\Phi_{1}$ is a largest left minorant of $\left\langle\operatorname{Re} \underline{\Omega^{-1}}\right\rangle$. If $\Omega(0)=I$, then $\Phi_{1}(0)=\Phi(0)$ immediately follows. Thus, part (a) is proved. Part (b) can be verified analogously

Theorem 3 leads us to the following interesting factorizations of maximal functions.

Proposition 6: The following statements hold true:

(a) Let $\Phi \in\left[H^{2}(\mathbb{D})\right]^{m \times m}$ be left maximal. Then there are an outer function $\varsigma \in$ $\left[\mathcal{N}_{+}(\mathrm{D})\right]^{m \times m}$ and a left maximal function $\varphi \in \mathcal{S}_{m \times m}(\mathrm{ID})$ such that $\Phi=\varsigma \varphi$. 
(b) Let $\Psi \in\left[H^{2} \cdot(\mathbb{D})\right]^{m \times m}$ be right maximal. Then there are an outer function $\eta \dot{\epsilon}$ $\left[\mathcal{N}_{+}(\mathbb{D})\right]^{m \times m}$ and a right maximal function $\psi \in \mathcal{S}_{m \times m}(\mathbb{D})$ such that $\Psi=\psi \eta$.

Proof: By the formula

$$
F(B):=\frac{1}{2 \pi} \int_{B} \underline{\Phi} \underline{\Phi}^{*} d \tilde{\lambda},
$$

where $B$ is any Borel subset of $\mathbf{T}$; a nonnegative Hermitian Borel measure $F$ on $\mathbf{T}$ is obviously given. By virtue of the matricial version of Herglotz' Theorem (see, e.g., [6, Theorem 2.2.2]) the function $\Omega: \mathbb{D} \rightarrow \mathbb{C}^{m \times m}$ defined by

$$
\Omega(z):=\int_{\mathbf{T}} \frac{\varsigma+z}{\varsigma-z} F(d \varsigma) \quad z \in \mathbb{D},
$$

belongs to $\mathcal{C}_{m}(\mathrm{ID})$. From Remark 6 and Lemma 4 we obtain $\left\langle\operatorname{Re} \Omega=\langle\underline{\Omega}\rangle \underline{\Phi}^{*}\right\rangle$. Since $\Phi$ is left maximal, $\Phi$ is a largest left minorant of $\langle\operatorname{Re} \underline{\Omega}\rangle$. Proposition 1 yields that $f:=(I-\Omega)(I+\Omega)^{-1}$ belongs to $\mathcal{S}_{m \times m}(\mathrm{DD})$ and that $\operatorname{det}(I+f)$ does not vanish in $\mathbb{D}$. Then we see from Proposition 2 that $(I+f)$ is an outer function which belongs to $\left[H^{\infty}(\mathrm{D})\right]^{\mathrm{m} \times m}$. Thus, Remark 2 provides that $\varsigma:=(I+f)^{-1}$ is an outer function in $\left[\mathcal{N}_{+}(\mathbb{D})\right]^{m \times m}$. By virtue of part (a) of Theorem 3, the $m \times m$ Schur function $\varphi:=2(I+\Omega)^{-1} \Phi$ is a largest left minorant of $\left\langle I-\underline{f} \underline{f}^{*}\right\rangle$. Proposition 1 yields that the identity (4) holds true. Hence, $\Phi=\frac{1}{2}(I+\Omega) \varphi \cdot=\varsigma \varphi$. Part (b) can be checked similarly

Observe that, for the operatorial case, a phenomenon of similar type as described in Proposition 6 was obtained by SUCIU and VALUSESCU [24, Theorem 8].

\section{On the Weyl Matrix Balls Associated with a Non- degenerate Matrix-valued Carathéodory Function}

Let $\tau$ be a nonnegative integer or $\tau=\infty$. A sequence $\left(\Gamma_{k}\right)_{k=0}^{\tau}$ of $m \times m$ complex matrices is called $m \times m$ Carathéodory sequence (respectively, nondegenerate $m \times m$ Carathéodory sequence) if, for every integer $n$ with $0 \leqq n \leqq \tau$, the block Toeplitz matrix

$$
T_{n}:=\operatorname{Re}\left[S_{n}\left\langle\Gamma_{0}, \Gamma_{1}, \ldots, \Gamma_{n}\right\rangle\right]
$$

where

$$
S_{n}\left(\Gamma_{0}, \Gamma_{1}, \ldots, \Gamma_{n}\right):=\left(\begin{array}{lllll}
\Gamma_{0} & 0 & 0 & \ldots & 0 \\
\Gamma_{1} & \Gamma_{0} & 0 & \ldots & 0 \\
\Gamma_{2} & \Gamma_{1} & \Gamma_{0} & \ldots & 0 \\
\vdots & \vdots & \vdots & \ddots & \vdots \\
\Gamma_{n} & \Gamma_{n-1} & \Gamma_{n-2} & \ldots & \Gamma_{0}
\end{array}\right),
$$

is nonnegative Hermitian (respectively, positive Hermitan). If $\left(\Gamma_{k}\right)_{k=0}^{\infty}$ is a given sequence of $m \times m$ complex matrices, then the power series

$$
\Omega(z):=\sum_{k=0}^{\infty} \Gamma_{k} z^{k}, \quad z \in \mathbb{D},
$$


defines an $m \times m$ Carathéodory function $\Omega$ if and only if $\left(\Gamma_{k}\right)_{k=0}^{\infty}$ is an $m \times m$ Carathéodory sequence (see, e.g., [8, Theorems 2.2.1 and 2.2.2]). An $m \times m$ Carathéodory function $\Omega$ is said to be nondegenerate if the sequence $\left(\Gamma_{k}\right)_{k=0}^{\infty}$ of its Taylor coefficients (in the Taylor series representation of $\Omega$ around the origin) is a nondegenerate $m \times m$ Caratheodory sequence.

Now we assume that $n$ is a nonnegative integer and that $\left(\Gamma_{k}\right)_{k=0}^{n}$ is a sequence of complex $m \times m$ matrices. We will use the notation $\mathcal{C}_{m}\left[\Gamma_{0}, \Gamma_{1}, \ldots, \Gamma_{n}\right]$ to denote the set of all $\Omega \in \mathcal{C}_{m}(\mathbb{D})$ for which $\left(\Gamma_{k}\right)_{k=0}^{n}$ is exactly the sequence of the first $n+1$ Taylor coefficients in the Taylor series representation of $\Omega$ around the origin. The set $\mathcal{C}_{m}\left[\Gamma_{0}, \Gamma_{1}, \ldots, \Gamma_{n}\right]$ is nonempty if and only if $\left(\Gamma_{k}\right)_{k=0}^{n}$ is an $m \times m$ Carathéodory sequence (see, e.g., [11, Part I, Section 4]). If $\left(\Gamma_{k}\right)_{k=0}^{n}$ is a nondegenerate Carathéodory sequence, then $\mathcal{C}_{m}\left[\Gamma_{0}, \Gamma_{1}, \ldots, \Gamma_{n}\right]$ can be described by certain linear fractional transformations (see, e.g., [11, Part V, Theorem 28]). Furthermore, in this case, one can show that, for each $z \in \mathbb{D}$, the set

$$
\left\{\Omega(z): \Omega \in \mathcal{C}_{m}\left[\Gamma_{0}, \Gamma_{1}, \ldots, \Gamma_{n}\right]\right\}
$$

can bc represented as a so-called matrix ball. If $M \in \mathbb{C}^{p \times q}, A \in \mathbb{C}^{p \times p}$ and $B \in \mathbb{C}^{q \times q}$, then the set

$$
\mathfrak{K}(M ; A, B):=\left\{X \in \mathbb{C}^{p \times q}: X=M+A K B, \mathbb{K} \in \mathbb{K}_{p \times q}\right\},
$$

where $\mathbb{K}_{p \times q}$ denotes the set of all $p \times q$ contractive matrices, is called the (closed) matrix ball with center $M$, left semi-radius $A$ and right semi-radius $B$. (In [23] SMMULJAN gave a summary of properties of matrix and operator balls.) In order to state the explicit representation of the set (25) as matrix ball, we need some preparations.

Suppose that $\left(\Gamma_{k}\right)_{k=0}^{n}$ is a nondegenerate $m \times m$ Caratheodory sequence. Then the matrices $\Gamma_{0}, \mathcal{G}_{n}:=S_{n}\left\langle\Gamma_{0}, \Gamma_{1}, \ldots, \Gamma_{n}\right\rangle$ and $T_{n}:=\operatorname{Re} \mathcal{G}_{n}$ are nonsingular. Set

$$
\begin{aligned}
z_{n}:=\left(\Gamma_{n}, \Gamma_{n-1}, \ldots, \Gamma_{1}\right), y_{n}:=\left(\Gamma_{1}^{*}, \Gamma_{2}^{*}, \ldots, \Gamma_{n}^{*}\right)^{*}, \\
l_{n}:= \begin{cases}\operatorname{Re} \Gamma_{0} & , n=0 \\
\operatorname{Re} \Gamma_{0}-\frac{1}{4} z_{n} T_{n-1}^{-1} z_{n}^{*}, & n>0\end{cases} \\
r_{n}:= \begin{cases}\operatorname{Re} \Gamma_{0} & n=0 \\
\operatorname{Re} \Gamma_{0}-\frac{1}{4} y_{n}^{*} T_{n-1}^{-1} y_{n}, & n>0\end{cases}
\end{aligned}
$$

and

$$
\mathfrak{T}_{n}:=\left(\mathfrak{S}_{n}^{-1}\right)^{*} T_{n} \mathfrak{S}_{n}^{-1} .
$$

Lemma 28 in [11, Part V] shows that $l_{n} \geqq 0$ and $r_{n} \geqq 0$. Furthermore, we define the matrix polynomials $\eta_{n}, \varsigma_{n}, \eta_{n}^{\prime}$ and $\varsigma_{n}^{\prime}$ by

$$
\begin{aligned}
& \eta_{n}(z):=e_{n m}(z) T_{n}^{-1} e_{n m}^{*}(0) \quad, \quad \varsigma_{n}(z):=\varepsilon_{n m}^{*}(0) T_{n}^{-1} \varepsilon_{n m}(z), \\
& \eta_{n}^{\prime}(z):=e_{n m}(z) \mathfrak{T}_{n}^{-1} e_{n m}^{*}(0) \quad, \quad \varsigma_{n}^{\prime}(z):=\varepsilon_{n m}^{*}(0) \mathfrak{T}_{n}^{-1} \varepsilon_{n m}(z),
\end{aligned}
$$

$z \in \mathbb{C}$, where $e_{n m}: \mathbb{C} \rightarrow \mathbb{C}^{m \times(n+1) m}$ and $\varepsilon_{n m}: \mathbb{C} \rightarrow \mathbb{C}^{(n+1) m \times m}$ are given by

$$
e_{n m}(z):=\left(I_{m}, z I_{m}, z^{2} I_{m}, \ldots, z^{n} I_{m}\right)
$$

and

$$
\varepsilon_{n m}(z):=\left(\bar{z}^{n} I_{m}, \bar{z}^{n-1} I_{m}, \ldots, \bar{z} I_{m}, I_{m}\right)^{*}, \quad z \in \mathbb{C}
$$


If $x_{0}, x_{1}, \ldots, x_{n}$ are complex $p \times q$ matrices, and if the $p \times q$ matrix polynomial $X$ is given by

$$
X(z):=\sum_{k=0}^{n} x_{k} z^{k}, \quad z \in \mathbb{C},
$$

then the reciprocal matrix polynomial $\tilde{X}$ of $X$ with respect to the unit circle $\mathbf{T}$ and the formal degree $n$ is the $q \times p$ matrix polynomial which is defined by

$$
\tilde{X}(z):=\sum_{k=0}^{n} x_{n-k}^{*} z^{k}, \quad z \in \mathbb{C} .
$$

In this sense, let $\tilde{\eta}_{n}$ (respectively, $\tilde{\eta}_{n}^{\prime}, \tilde{\zeta}_{n}, \tilde{\zeta}_{n}^{\prime}$ ) be the reciprocal matrix polynomial of $\eta_{n}$ (respectively, $\eta_{n}^{\prime}, \varsigma_{n}, \varsigma_{n}^{\prime}$ ) with respect to $\mathbf{T}$ and the formal degree $n$. One can check that the matrices

$$
P(z):=\varsigma_{n}^{*}(z) l_{n} \varsigma_{n}(z)-|z|^{2}\left(\tilde{\eta}_{n}(z)\right)^{*} r_{n} \tilde{\eta}_{n}(z)
$$

and

$$
Q(z):=\eta_{n}(z) r_{n} \eta_{n}^{*}(z)-|z|^{2} \tilde{\zeta}_{n}(z) l_{n}\left(\tilde{\zeta}_{n}(z)\right)^{*}
$$

are positive Hermitian for all $z \in \mathbb{D}$ (see [11, Part $V$, Theorem 29]). The functions $\mathfrak{M}_{n}: \mathbb{D} \rightarrow \mathbb{C}^{m \times m}, \mathfrak{L}_{n}^{\#}: \mathbb{D} \rightarrow \mathbb{C}^{m \times m}$ and $\mathfrak{R}_{n}: \mathbb{D} \rightarrow \mathbb{C}^{m \times m}$ given by

$$
\begin{aligned}
& \mathfrak{N}_{n}(z):=\left[\eta_{n}^{\prime}(z)\left(\Gamma_{0}^{-1}\right)^{*} r_{n} \eta_{n}^{*}(z)+|z|^{2} \tilde{\varsigma}_{n}^{\prime}(z) \Gamma_{0}^{-1} l_{n}\left(\tilde{\zeta}_{n}(z)\right)^{*}\right][Q(z)]^{-1}, \\
& \mathfrak{L}_{n}^{\#}(z):=[P(z)]^{-1} \text { and } \mathfrak{R}_{n}(z):=[Q(z)]^{-1}
\end{aligned}
$$

are called the Weyl-Carathéodory center function, the canonical normalized left WeylCarathéodory semi-radius function and the canonical right Weyl-Carathéodory semiradius function, respectively, associated with the nondegenerate $m \times m$ Carathéodory sequence $\left(\Gamma_{k}\right)_{k=0}^{n}$. One can show that the inequalities

$$
\operatorname{Re} \mathfrak{M}_{n}(z) \geqq 0, \mathcal{L}_{n}^{\#}(z)>0 \text { and } \Re_{n}(z)>0
$$

hold true for every choice of $z$ in $\mathrm{D}$ (see, e.g., [13, Lemma 6]).

Now we are able to state the announced representation of the set.(25) as matrix ball. A proof of this result can be found in [11, Part V, Theorem 29].

Theorem 5: Let $n \in \mathbb{N}_{0}$, and let $\left(\Gamma_{k}\right)_{k=0}^{n}$ be a nondegenerate $m \times m$ Carathéodory sequence. Further, let $\mathfrak{M}_{n}, \mathfrak{L}_{n}^{\#}$ and $\mathfrak{R}_{n}$ be the Weyl-Carathéodory center function, the canonical normalized left and the canonical right Weyl-Carathéodory semi-radius functions, respectively, associated with $\left(\Gamma_{k}\right)_{k=0}^{n}$. For each $z \in \mathbb{D}$, the set $(25)$ coincides with the matrix ball $\mathfrak{K}\left(\mathfrak{M}_{n}(z) ;|z|^{n+1} \sqrt{2 \mathfrak{L}_{n}^{\#}(z)}, \sqrt{2 \mathfrak{R}_{n}(z)}\right)$.

Now we turn our attention to the limit behaviour of the Weyl matrix balls associated with a nondegenerate $m \times m$ Carathéodory function.

Theorem 6 (see [11, Theorem 4]): Let $\Omega$ bè a nondegenerate $m \times m$ Carathéodory function, and let (8) be the Taylor series representation of $\Omega$. For $n \in \mathbb{N}_{0}$, let $\mathfrak{M}_{n}$, $\mathfrak{L}_{n}^{\#}$ and $\mathfrak{R}_{n}$ be the Weyl-Carathéodory center function, the canonical normalized left and the canonical right Weyl-Carathéodory semi-radius functions, respectively, associated with $\left(\Gamma_{k}\right)_{k=0}^{n}$. Then: 
(a) For each $z \in \mathbb{D}, \lim _{n \rightarrow \infty} \mathfrak{M}_{n}(z)=\Omega(z)$.

(b) For each $z \in \mathbb{D}$, the sequences $\left(\mathcal{L}_{n}^{\#}(z)\right)_{n=0}^{\infty}$ and $\left(\Re_{n}(z)\right)_{n=0}^{\infty}$ are monotonously nonincreasing and convergent. The corresponding limits $\mathfrak{L}^{\#}(z)$ and $\mathfrak{R}(z)$ are nonnegative Hermitian for all $z \in \mathbb{D}$.

Observe that parts of Theorems 5 and 6 are already contained in KOVALISHINA's paper [17].

The functions $\mathfrak{L}^{\#}: \mathbb{D} \rightarrow \mathbb{C}^{m \times m}$ and $\Re: \mathbb{D} \rightarrow \mathbb{C}^{m \times m}$ given in part (b) of Theorem 6 are called the canonical normalized left and the canonical right Weyl-Carathéodory limit semi-radius functions, respectively, associated with the nondegenerate $m \times m$ Carathéodory function $\Omega$.

\section{Factorizations of the Normalized Limit Semi-radii Functions Associated with a Matrix-valued Cara- théodory Function with Finite Entropy}

Let us begin this section with some technical preparations.

Lemma 5: Let $\Omega \in \mathcal{C}_{m}$ (II)). If

$$
\frac{1}{2 \pi} \int_{\boldsymbol{T}} \log [\operatorname{det}(\operatorname{Re} \underline{\Omega})] d \tilde{\lambda}>-\infty,
$$

then $\Omega$ is necessarily nondegenerate.

Proof: Combine Corollary 3 in [11, Part II] and Lemma 4

Functions $\Omega \in \mathcal{C}_{m}$ (ID) which satisfy inequality (33) are called $m \times m$ Carathéodory functions of finite entropy. In the following we will only consider Carathéodory functions of that type.

Note that the proof of the following proposition is essentially based on results on the asymptotic behaviour of orthogonal matrix polynomials.

Proposition 7: Let $\Omega$ be an $m \times m$ Carathéodory function which satisfies condition (33), and let (8) be the Taylor series representation of $\Omega$. For $n \in \mathbb{N}_{0}$, let the matrix polynomials $\eta_{n}$ and $\varsigma_{n}$ be defined by (22), (28), (29) and (26). Then:

(a) For all $z \in \mathbb{D}$,

$$
\lim _{n \rightarrow \infty} \tilde{\eta}_{n}(z)=0 \text { and } \lim _{n \rightarrow \infty} \tilde{\varsigma}_{n}(z)=0
$$

(b) For each $z \in \mathbb{D}$, there exist the limits $\eta(z):=\lim _{n \rightarrow \infty} \eta_{n}(z)$ and $\varsigma(z):=\lim _{n \rightarrow \infty} \varsigma_{n}(z)$.

(c) The matrices $\eta(0)$ and $\varsigma(0)$ are positive Hermitian.

(d) For all $z \in \mathbb{D}$, det $\eta(z)=\operatorname{det} \varsigma(z) \neq 0$. 
(e) The matrix-valued functions $\Phi_{0}: \mathbb{D} \rightarrow \mathbb{C}^{m \times m}$ and $\Psi_{0}: \mathbb{D} \rightarrow \mathbb{C}^{m \times m}$ given by

$$
\Phi_{0}(z):=[\varsigma(z)]^{-1} \sqrt{\varsigma(0)} \text { and } \Psi_{0}(z):=\sqrt{\eta(0)}[\eta(z)]^{-1}
$$

are outer functions in $\left[H^{2}(\mathbb{D})\right]^{m \times m}$.

(f) The function $\Phi_{0}$ is the normalized outer left spectral factor of $\langle\operatorname{Re} \Omega\rangle$, whereas $\Psi_{0}$ is the normalized outer right spectral factor of $(\operatorname{Re} \underline{\Omega})$.

Proof: From Lemma 5 we know that $\Omega$ is nondegenerate. Let $F$ be the F.-RieszHerglotz measure associated with $\Omega$. In view of (22) and Remark 6, we have

$$
T_{n}=\operatorname{Re}\left(S_{n}\left\langle C_{0}^{(F)}, C_{1}^{(F)}, \ldots, C_{n}^{(F)}\right\rangle\right) .
$$

Furthermore, we know from Lemma 4 that Re $\Omega$ is the Radon-Nikodym derivative of the absolutely continuous part of $F$ in the Lebesgue decomposition of $F$ with respect to $\tilde{\lambda}$. Thus, parts (b) - (f) follow inmediately from results due to DELSARTE, GENIN and KAMP [6, Theorems 17, 18 and 20]. It remains to prove part (a). By virtue of Lemma 3.6 .3 in [8] and formula (88) in [6], we have

$$
\left[\Psi_{0}(z)\right]^{-1}\left(\left[\Psi_{0}(z)\right]^{-1}\right)^{*}=\left(1-|z|^{2}\right) \sum_{k=0}^{\infty} \tilde{\varsigma}_{n}(z)\left[\varsigma_{n}(0)\right]^{-1}\left[\tilde{\varsigma}_{n}(z)\right]^{*}
$$

for all $z \in \mathbb{D}$. This implies $\lim _{n \rightarrow \infty}\left(\tilde{\zeta}_{n}(z){\sqrt{\varsigma_{n}(0)}}^{-1}\right)\left(\tilde{\zeta}_{n}(z){\sqrt{\varsigma_{n}(0)}}^{-1}\right)^{*}=0$, and hence $\lim _{n \rightarrow \infty} \tilde{\zeta}_{n}(z){\sqrt{\varsigma_{n}(0)}}^{-1}=0$. Thus, we can conclude from parts (b) and (d) that the second relation in (34) holds true. The first one follows analogously

Note if $f$ is a matrix-valued Schur function, then several results on polynomial approximation of appropriate outer spectral factors of $\left\langle I-\underline{f} \underline{f}^{*}\right\rangle$ and $\left\langle I-\underline{f}^{*} \underline{f}\right\rangle$ are scattered in the literature (see DUBOVOJ [7], GEORGIOU and K $\overline{\mathrm{H} A R G O N E K A} \overline{\mathrm{R}}$ [14] and, for the operator case, BAKONYI [3]).

Now we are able to derive the announced factorizations of the canonical normalized Weyl-Carathéodory limit semi-radius functions associated with a matrix-valued Carathéodory function having finite entropy.

Theorem 7: Let $\Omega$ be an $m \times m$ Carathéodory function which satisfies condition (33). Let $\mathfrak{L}^{\#}$ and $\Re$ be the canonical normalized left and the canonical right Weyl-Carathéodory limit semi-radius functions, respectively, associated with $\Omega$.

(a) Let $\Phi$ be an arbitrary largest lefi minorant of $\langle\operatorname{Re} \underline{\Omega}\rangle$. Then $\Phi \Phi^{*}=\mathfrak{L}^{\#}$.

(b) Let $\Psi$ be. an arbitrary largest right minorant of $\langle\operatorname{Re} \underline{\Omega}\rangle$. Then $\Psi^{*} \Psi=\Re$.

Proof: Lemma 5 shows that $\Omega$ is nondegenerate. By virtue of part (f) of Proposition $7, \Phi_{0}$ (respectively, $\Psi_{0}$ ) given in (35) is a left (respectively, right) spectral factor of $\langle\operatorname{Re} \Omega\rangle$. For all positive integers $n$, we see from Lemma 3.6.33 in [8] that $\eta_{n}(0)=r_{n}^{-1}>0$ and 
$\varsigma_{n}(0)=l_{n}^{-1}>0$. Ujsing Proposition 7 we get then $\lim _{n \rightarrow \infty} r_{n}=[\eta(0)]^{-1}, \lim _{n \rightarrow \infty} l_{n}=$ $[\varsigma(0)]^{-1}$, and hence

$$
\lim _{n \rightarrow \infty}\left(\varsigma_{n}^{*}(z) l_{n} \varsigma_{n}(z)-|z|^{2}\left[\tilde{\eta}_{n}(z)\right]^{*} r_{n} \tilde{\eta}_{n}(z)\right)=\varsigma^{*}(z)[\varsigma(0)]^{-1} \varsigma(z)>0 .
$$

In view of (35) this implies $\lim _{n-\infty} \mathfrak{L}_{n}^{\#}(z)=\Phi_{0}(z) \Phi_{0}^{*}(z)$ for every choice of $z \in$ ID. Analogously, we obtain $\lim _{n \rightarrow \infty} \mathfrak{R}_{n}(z)=\Psi_{0}^{*}(z) \Psi_{0}(z)$ for each $z \in \mathbb{D}$. Theorem 2 shows that there are $m \times m$ unitary matrices $U$ and $V$ such that $\Phi=\Phi_{0} U$ and $\Psi=V \Psi_{0}$. Thus, the assertion immediately follows

Now we will sketch the way how Theorem 7 can be used to refound the factorizations of the limit semi-radii of the Weyl matrix balls associated with a matrix-valued Schur function, which the authors obtained in [12, Part IV].

Assume that $f$ is a $p \times q$ Schur function which satisfies

$$
\frac{1}{2 \pi} \int_{\mathbf{T}} \log \operatorname{det}\left(I-\underline{f} \underline{f}^{*}\right) d \tilde{\lambda}>-\infty .
$$

Then there are outer functions $\varphi \in\left[H^{2}(\mathbb{D})\right]^{p \times p}$ and $\psi \in\left[H^{2}(\mathbb{D})\right]^{q \times q}$ such that

$$
I-\underline{f} \underline{f}^{*}=\underline{\varphi} \underline{\varphi}^{*} \quad \text { and } \quad I-\underline{f}^{*} \underline{f}=\underline{\psi}^{*} \underline{\psi} \quad \tilde{\lambda} \text {-a.e. on } \mathbf{T}
$$

(see Theorem 2). Let

$$
f(z)=\sum_{k=0}^{\infty} A_{k} z^{k}, \quad z \in \mathbb{D},
$$

be the Taylor series representation of $f$. For every nonnegative integer $n$, then the symbol $S_{p \times q}\left[A_{0}, A_{1}, \ldots, A_{n}\right]$ stands for the set of all $g \in \mathcal{S}_{p \times q}(\mathrm{D})$ with first $n+1$ Taylor coefficients $A_{0}, A_{1}, \ldots, A_{n}$ in the Taylor series representation of $g$ around the origin. Then for each $z \in \mathbb{D}$, the set $\left\{g(z): g \in \mathcal{S}_{p \times q}\left[A_{0}, A_{1}, \ldots, A_{n}\right]\right\}$ turns out to be a matrix ball

$$
\mathfrak{K}\left(\mathcal{M}_{n}(z) ;|z|^{n+1} \sqrt{\mathcal{L}_{n}^{\#}(z)}, \sqrt{\mathcal{R}_{n}(z)}\right)
$$

where $\mathcal{L}_{n}^{\#}(z)$ and $\mathcal{R}_{n}^{\prime}(z)$ are positive Hermitian matrices with $\operatorname{det} \mathcal{L}_{n}^{\#}(z)=\operatorname{det} \mathcal{R}_{n}(z)$ (see, e.g., $\left[8\right.$, Theorem 5.5.1, Lemma 5.6.3]). For each $z \in \mathbb{D}$, the sequences $\left(\mathcal{L}_{n}^{\#}(z)\right)_{n=0}^{\infty}$ and $\left(\mathcal{R}_{n}(z)\right)_{n=0}^{\infty}$ are monotonously nonincreasing and hence convergent to some nonnegative Hermitian matrices $\mathcal{L}^{\#}(z)$ and $\mathcal{R}(z)$, respectively. Part (d) of Theorem 18 in $[12$, Part IV] yields that

$$
\mathcal{L}^{\#}(z)=\varphi(z) \varphi^{*}(z) \quad \text { and } \quad \mathcal{R}(z)=\psi^{*}(z) \psi(z)
$$

for all $z \in \mathbb{D}$. This result proves to be a special case of Theorem 7 . One has only to consider the particular $(p+q) \times(p+q)$ Carathéodory function $\Omega$ given by

$$
\Omega(z):=\left(\begin{array}{cc}
I_{p} & -2 z f(z) \\
0 & I_{q}
\end{array}\right), \quad z \in \mathbb{D} .
$$

Using the notations given above one obtains $\mathfrak{L}^{\#}=\operatorname{diag}\left(\mathcal{L}^{\#}, I_{q}\right)$ and $\mathfrak{R}=\operatorname{diag}\left(I_{p}, \mathcal{R}\right)$. We omit the lengthy, but straightforward computations. 


\section{References}

[1] ACHIEsER, N.I.: On weighted approximation of continuous functions by polynomials on the entire real axis (in Russian). Uspehi Mat. Nauk 11 (1956), 4, 3 - 43. Engl. transl.: Amer. Math. Soc. Transl. (2) 22 (1962), $95-137$.

[2] ARov, D.Z.: $\gamma$-generating matrices, $J$-inner matrix-functions and related extrapolation problems (in Russian). Teor. Funkcii, Funkt. Anal. Pril., Part I: 51 (1989), 61 - 67; Part II: 52 (1989), 103 109; Part III: 53 (1990), 57 - 64.

[3] Bakonyı, M.: Spectral factors and analytic completions. Int. Equ. Oper. Theory 13 (1990), 149 164.

[4] BRodSKII, M.S.: Unitary operator colligations and their characteristic functions (in Russian). Uspehi Mat. Nauk 33 (1979) (4), 144 - 168. Engl. transl.: Russ. Math. Surveys 22 (1978), 159 191.

[5] Constantinescu, T.: On a general extrapolation problem. Rev. Roumaine Math. Pures Appl. 32 (1987), $509-521$

[6] Delsarte, P., Genin, Y., and Y. KamP: Othogonal polynomial matrices on the unit circle. IEEE Trans. Circ. Syst. CAS - 25 (1978), $145-160$.

[7] Dubovos, V.K.: Indefinite metric in the interpolation problem of Schur for analytic matrix functions, Part V. (in Russian). Teor. Funkcii, Funkt. Anal. Pril. 45 (1986), 16 - 26

[8] Dubovoj, V.K., Fritzsche, B., and B. Kirstein: Matricial Version of the Classical Schur Problem, (Teubner-Texte zur Mathematik: Band 129). Stuttgart-Leipzig: B.G. Teubner Verlagsges. 1992.

[9] Dubovoj, V.K., and Ramadan K. Mohammed: Defect functions of holomorphic contractive matrix functions, regular extensions and open systems. Math. Nachr. (to appear).

[10] Duren, P.L.: Theory of $H^{p}$ Spaces. New York: Academic Press 1970.

[11] FRITZSCHE, B., and B. KiRstein: An extension problem for non-negative Hermitian block Toeplitz matrices. Math. Nachr., Part I: 130 (1987), 121 - 135; Part II: 131 (1987), 287 - 297; Part III: 135 (1988), 319 - 341; Part IV: 143 (1989), 329 - 354; Part V: 144 (1989), 283 - 308.

[12] Fritzsche, B., and B. Kirstein: A Schur type matrix extension problem. Math. Nachr., Part 1: 134 (1987), 257 - 271; Part II: 138 (1988), 195 - 216; Part III: 143 (1989), 227 - 247; Part IV: 147 (1990), 235 - 258

[13] Fritzsche, B., and B. KiRstein: On the Weyl matrix balls associated with nondegenerate matrixvalued Corathéodory functions. Z. Anal. Anw. (to appear).

[14] GeORgIOU, T.T., and P.P. KHARgONEKAR: Spectral factorization of matrix-valued functions using interpolation theory. IEEE Trans. Circ. Syst. CAS - 36 (1989), 568 - 574.

[15] Helson H., and D. Lowdenslager: Prediction theory and Fourier series in several variables. Acta Math., Part I: 99 (1958), 162 - 202; Part II: 106 (1961), 175 - 213.

[16] Katsnelson, V.E.: Extremal and factorization properties of the radii in the problem of representation of Hermitian-positive functions (in Russian). In: Mathematical Physics and Functional Analysis (Ed.: V.A. MARĈENKO). Kiev: Naukova Dumka 1986, pp. 80 - 94.

[17] Kovalishina, I.V.: Analytic theory of a class of interpolation problems (in Russian). Izv. Akad. Nauk SSSR, Ser. Mat., 47 (1983), 455 - 497. Engl. transl.: Math. USSR Izvestija 22 (1984), 419 463.

[18] KREIN, M.G.: On the continuation problem for Hermitian-positive continuous functions (in Russian). Dokl. Akad. Nauk SSSR 26 (1940), 17 - 21.

[19] MASANI, P.R.: Cramer's theorem on monotone matrix-valued functions and the Wold decomposition. In: Probability and Statistics - The Harald Cramér Volume (Ed.: U. Grenander). Stockholm: Almquist \& Wiksell 1959, pp. 175 - 189. 
[20] Masani, P.R.: Shift invariant spaces and prediction theory. Acta Math. 107 (1962), 275 - 290.

[21] Mergeljan, S.N.: Weighted approximation by polynomials (in Russian). Uspehi Math. Nauk 11 (1956) (3), 107 - 152. Engl. transl.: Amer. Math. Soc. Transl. (2) 10 (1958), 59 - 106

[22] Nevandinna, R.: Eindeutige analytische Funktionen. Berlin: Springer-Verlag 1953.

[23] S̆muljan, J.L.: Operator balls (in Russian). Teor. Funkcii, Funkt. Anal Pril. 6 (1968), 68 - 81. Engl. transl.: Int. Equ. Oper. Theory 13 (1990), $864-882$.

[24] Suciu, I., and I. Valusescu: Factorization theorems and prediction theory. Rev. Roumaine Math. Pures Appl. 23 (1978), 1393 - 1423.

[25] Sz.-NAGY, B., and C. FolAS: Harmonic Analysis of Operators in Hilbert Space. Budapest: Akademiai Kiadó 1970 .

[26] WIEnER, N., and P.R. MASANI: The prediction theory of multivariate stochastic processes. Acta Math., Part I: 98 (1957), 111 - 150; Part II: 99 (1958), 93 - 137. 